University of Nebraska - Lincoln

DigitalCommons@University of Nebraska - Lincoln

CSE Conference and Workshop Papers

Computer Science and Engineering, Department

2012

\title{
Mobile Data Harvesting in Wireless Underground Sensor Networks
}

John Tooker

University of Nebraska-Lincoln, jtooker@cse.unl.edu

Mehmet C. Vuran

University of Nebraska-Lincoln, mcvuran@cse.unl.edu

Follow this and additional works at: https://digitalcommons.unl.edu/cseconfwork

Part of the Computer Sciences Commons

Tooker, John and Vuran, Mehmet C., "Mobile Data Harvesting in Wireless Underground Sensor Networks" (2012). CSE Conference and Workshop Papers. 205.

https://digitalcommons.unl.edu/cseconfwork/205

This Article is brought to you for free and open access by the Computer Science and Engineering, Department of at DigitalCommons@University of Nebraska - Lincoln. It has been accepted for inclusion in CSE Conference and Workshop Papers by an authorized administrator of DigitalCommons@University of Nebraska - Lincoln. 


\title{
Mobile Data Harvesting in Wireless Underground Sensor Networks
}

\author{
John Tooker Mehmet C. Vuran \\ Cyber-Physical Networking Laboratory \\ Department of Computer Science and Engineering \\ University of Nebraska-Lincoln, Lincoln, NE 68588 \\ Tel: (402) 472-5019 Fax: (402) 472-7767 \\ Email: \{jtooker,mcvuran\}@ cse.unl.edu
}

\begin{abstract}
Wireless Underground Sensor Networks (WUSNs) allow for continuous field monitoring without interfering with aboveground activities, such as plowing or football games. Due to the increased path loss in soil, it is challenging to ensure that a large-scale underground network is connected while still being cost effective in terms of deployment and maintenance.

In this paper, a practical WUSN architecture is developed, consisting of mobile nodes that harvest data from stationary underground nodes. To this end, the impacts of packet size and error control schemes on network performance are investigated through field experiments. By developing a better understanding of the wireless channel and the reliability between underground and aboveground nodes, a family of mobile WUSN protocols is developed and evaluated in terms of packet delivery success, delay tolerance and network lifetime.
\end{abstract}

\section{INTRODUCTION}

Wireless sensor networks (WSNs) seek to monitor an environment and report events in a cheap, reliable manner [1]. Motes are small, low-cost computers equipped with microprocessors, low power radios and sensing devices. Hundreds (or thousands) of devices are used to monitor an environment and relay data through a network. Wireless underground sensor networks (WUSNs) extend sensing and transmission into the soil. WUSNs monitor soil properties while lying under the surface where they are safe and out of the way of aboveground activities [2]. This paper investigates a scalable WUSN architecutre, which achieves a long network lifetime by using mobile nodes to harvest data collected by underground nodes.

The scalability and network lifetime of a WUSN are limited by energy and communication range. Being underground (UG), motes cannot replenish energy, nor is it cost-feasible to frequently dig up motes to replace batteries [3]. Due to the short communication range in the UG channel, two UG motes must be within one to five meters of each other to communicate [2]. This yields a high density of UG motes, which is also not cost-feasible [4].

As shown in Fig. 1, mobile sinks, however, can be used to connect a sparsely deployed network of stationary sensor nodes [4], [5]. Thus, an UG node is connected to the network if a mobile sink collects its data in a certain time frame. The limit of this time frame, also known as the delay tolerance of the network, determines how many mobile nodes are needed.

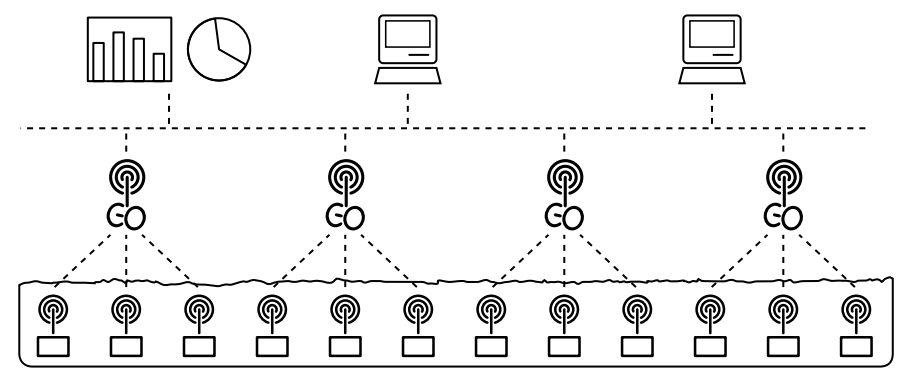

Fig. 1. Network architecture of the proposed WUSN.

Mobile sinks are ideal for delay tolerant applications of WUSNs, such as agricultural field monitoring and sports field maintenance. As agriculture makes up $1.2 \%$ of the United States' economy and $6 \%$ of the world's economy [6], improvements in crop efficiency have a large impact on the world. Continuous monitoring of soil properties (moisture, $\mathrm{pH}$ balance) give farmers detailed recommendations on how to irrigate and fertilize. Today, existing precision agriculture tools rely on soil data collected in a non-continuous way, often once a year [7]. Field monitoring is the target application of the mobile, data harvesting WUSNs modeled and evaluated herein.

Terrestrial WSNs may also be used to monitor field conditions [8], but require permanent aboveground (AG) devices. WUSNs have no permanent AG fixtures to get in the way of AG activities, such as crop harvesting. Therefore, with WUSNs, the field can be used while soil data is continuously collected.

WUSNs, however, must transmit data through the soil. There are three different UG channels to consider [9]:

- Underground-to-Underground Channel (UG2UG)

- Aboveground-to-Underground Channel (AG2UG)

- Underground-to-Aboveground Channel (UG2AG)

Each of these channels exhibits different characteristics related to wireless transmission. The range of AG2UG and UG2AG communication is much greater than UG2UG, ranging from $5 \mathrm{~m}$ to $80 \mathrm{~m}$ versus $1 \mathrm{~m}$ to $5 \mathrm{~m}$, as shown in [2], [9] and recent experiments. In general the UG2AG channel is better than the AG2UG channel, thus communication is asymmetric. 
Soil moisture heavily affects the communication through soil in each of these channels [2].

Packet size and error control also play a large role in wireless transmission [10]. Longer packets reduce the ratio of overhead to payload, but longer packets are more susceptible to errors. Error control protocols, such as automatic retry request (ARQ) or forward error correction (FEC) are able to achieve higher packet reception rates (PRRs) than using no error control at all. As the focus on underground communication has so far been on channel modeling, these protocols have not been empirically evaluated yet. To this end, experiments were run to gain detailed information about the link layer properties of WUSNs. This data is necessary to accurately evaluate the WUSN models proposed in Section IV.

The remainder of this paper is organized as follows. In Section II, work related to WUSNs, including the underground channels, is presented. Experiments to characterize the impacts of link layer parameters on UG2AG and AG2UG channels are presented and evaluated in Section III. In Section IV, a WUSN model is proposed, along with various communication protocols. This model is evaluated in Section V using the empirical results collected in Section III. This paper is concluded in Section VI.

\section{RELATED WORK}

The UG channel model is explored in [2], [11]. The moisture of the soil has a large impact on bit error rate. Soil with a 5\% volumetric water content (VWC) can achieve bit error rates under $0.01 \%$, while soil with a VWC of $15 \%$ loses $1 \%$ of transmitted bits. Thus, on days after rain, when the soil moisture is over $20 \%$, UG communication is severely limited. While focusing on the UG2UG channel, it is concluded in [2] that multi-hop communication is needed for WUSNs. However, this implies having a density of many nodes per square meter. Such an architecture may lead to costs that are not feasible for a large (agricultural-sized) field.

The communication range is an order of magnitude larger for the UG2AG and AG2UG channels as compared to the UG2UG channel [9]. Thus, AG mobile nodes may be used to connect a WUSN. In a sparse WUSN, data would be buffered at each UG mote. Thus, sending longer packets would increase throughput. There is a limit, however to how long packets should be, as longer packets are more susceptible to bit errors [12]. As UG channel models are still being developed, empirical results are needed to confirm UG channel theory, as presented in [3], [4], [9]-[12].

A mobile sink WSN paradigm uses data MULEs (exclusively) to collect data [5]. Every packet is only transmitted once, from the sensor mote to the MULE, no mesh network is formed. Relay nodes need not exist, only enough static nodes to detect events are deployed. This type of network, however, suffers from a higher latency than a multi-hop network. So far, there has been no effort to exclusively extend this type of network underground, though the work presented in [4] uses mobile sinks to supplement connectivity.
Connectivity of a WUSN is discussed in [4]. A WUSN model consisting of a field with many UG nodes in its interior with multiple stationary AG sinks at its border is presented. UG nodes use multi-hop communication to relay data to the edge of the field. In this architecture, mobile sinks are assumed to move over the field to supplement information gathering as there may be pockets of UG nodes that are interconnected without being connected to a single sink. An UG node is connected to the network if it can either transmit its packet to another connected UG node or if it can transmit to a sink (mobile or stationary) within a given time limit [4]. This model requires a sensor density of 1 node for every $4 \mathrm{~m}^{2}$ to achieve a high connection probability. This is not cost effective as 40,000 motes are needed for a 40 acre field. In addition, to the best of our knowledge, network scalability, lifetime and energy consumption in WUSNs have not been discussed or evaluated yet.

\section{EMPIRICAl ANALYSis of PACKET Size AND ERROR CONTROL ON UG TO AG COMMUNICATION}

In this section, the effects of packet size and error control protocols on the UG2AG and AG2UG channels are investigated for use in evaluating the WUSN protocols proposed in Section IV.

The effects of packet size and error control scheme are key as the throughput of a node in a WUSN strongly depends on packet size and its corresponding packet reception rate (PRR) [10]. UG channel models are still in development. Empirical results are needed to supplement UG channel theory, as presented in [3], [4], [9]-[12]. These results will be used in the evaluation of the WUSN protocols in Section IV.

\section{A. Experimental Setup}

In the following experiments, an underground mote exchanged packets of various sizes with an aboveground node. Mica2 motes were used for these experiments as they operate at $433 \mathrm{MHz}$, a lower frequency than TelosB or MicaZ motes, which penetrates the soil better [9]. Both ARQ and FEC error control protocols were evaluated. For the ARQ protocol, latency was measured from the time the first data packet was attempted until an ACK was received.

The Mica 2 mote and its CC1000 radio allow for arbitrarily large packets to be transmitted as well as access to packets that are received with bits in error. This allows for packet sizes of up to $1 \mathrm{kB}$ to be used in experiments. As FEC was not supported in hardware, it was simulated in software. Two levels of error detection were employed. The first was a CRC check that could detect bit errors in a packet. The second level could correct bit errors in a packet's payload (up to 200 bits in error). This was possible as payloads were deterministic and known by the receiver. This results in a software-implemented FEC protocol and provides a lower bound on the effectiveness of such protocols.

For the experiments, a full wave antenna was used for the AG mote, which was suspended horizontally $30 \mathrm{~cm}$ above the soil [13]. A planar disc antenna was used for the UG mote 


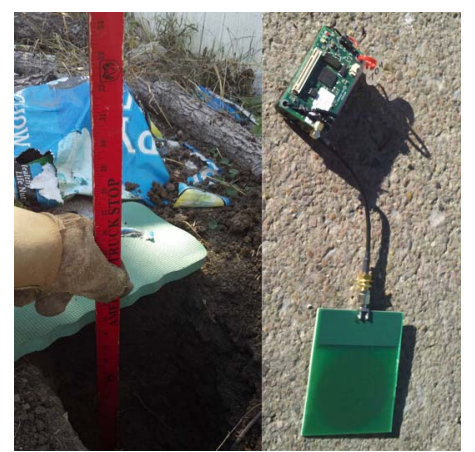

(a)

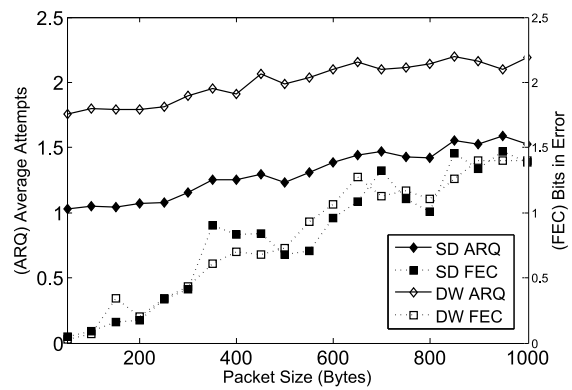

(b)

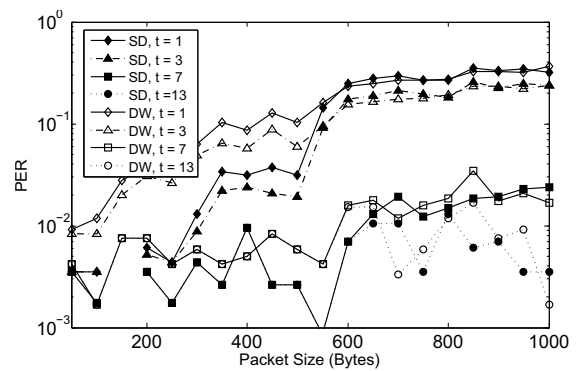

(c)

Fig. 2. (a) Pictures of the experiments, (b) Average attempts (ARQ) and bits in error (FEC), and (c) PER of various FEC encryption strengths.

and was buried directly under the AG mote. These antennae were chosen as they outperformed dipole antennae [13]. Photographs from the experiment can be seen in Fig. 2(a). The experiments were performed in three different configurations:

1) Shallow-deep (SD): The UG mote was buried at a depth of $30 \mathrm{~cm}$ and the experiments were performed when the ground was dry (volumetric water content (VWC) $\sim 5 \%)$.

2) Deep-wet (DW): The UG node was buried $50 \mathrm{~cm}$ deep and the experiments were performed when the ground was wet (VWC $\sim 20 \%$ ).

3) Shallow-wet (SW): While the soil moisture was $24 \%$, the mote was buried at a depth of $30 \mathrm{~cm}$.

During the 76 hours of the SD experiment, 1,144 packets were sent for each data point. Only $0.38 \%$ of packets were not reported or were corrupt. These were pruned before analyzing the data. 1,192 packets were sent for each data point in the DW experiment. Only $0.27 \%$ of packets were not reported or were corrupt in the data collected. These were pruned before analysis. In the SW experiment, 1,070 packets were sent for each data point. Only $0.40 \%$ of packets were not reported or were corrupt, and were pruned as well.

In the protocols used, ARQ packets required an acknowledgment to be received for it to be marked as successfully delivered, while FEC packets did not. Up to five retries were performed before dropping the packet using the ARQ protocol. If not stated, the error correction capability of the FEC protocol, $t$, was seven bits using a BCH Code $(t=7$ with $k$ and $n$ dependent on the packet size), other strengths were also evaluated. In the results shown, experiment results for the UG2AG channel are considered, unless otherwise specified.

\section{B. Underground Experiment Results}

The average signal-to-noise ratio (SNR) between the UG node and the AG node for each experiment run is shown in Table I. The added depth and moisture decreased the SNR value for the deep experiment by $14 \mathrm{~dB}$ in each direction as compared to the shallow values. Packets in the UG2AG channel were received $14 \mathrm{~dB}$ better than AG2UG, except for the SW experiment, where the difference was only $4.5 \mathrm{~dB}$. This trend is consistent with the previous work [4], [9], [13].
TABLE I

AVERAGE SNR VALUES FROM THE UG2AG AND AG2UG EXPERIMENTS

\begin{tabular}{llll}
\hline & SD & SW & DW \\
\hline AG2UG & $52.37 \mathrm{~dB}$ & $29.45 \mathrm{~dB}$ & $38.08 \mathrm{~dB}$ \\
UG2AG & $38.02 \mathrm{~dB}$ & $25.00 \mathrm{~dB}$ & $24.90 \mathrm{~dB}$ \\
\hline
\end{tabular}

The SW experiment, had the worse AG2UG channel quality, though its UG2AG channel was similar to that of the DW experiment. In the following, the results for SD and DW experiments are discussed as they represent extreme cases in UG communication. SW experiment results are also shown where necessary.

In Fig. 2(b), the effects of packet size on the ARQ and FEC protocols are shown. For the ARQ protocol, the average number of attempts are shown (left y-axis), whereas for the FEC protocol, the number of bits in error corrected by the FEC protocol are shown (right y-axis). Both depend on packet size and soil condition. Small packets (50 Bytes) in SD conditions are received with no bits in error. However, large packets $(1 \mathrm{kB})$ in $\mathrm{SD}$ conditions required, on average, 1.5 attempts for the ARQ protocol and the FEC protocol must correct 1.5 bits. The average number of bits in error for FEC was not affected by changes in channel conditions, but compared to $\mathrm{SD}$, the number of attempts of the ARQ protocol increased by 0.75 for DW, for all packet sizes.

The effect of FEC error correction capability, $t$, on the packet error rate (PER) is shown in Fig. 2(c). For $t<7$ and packets under 500 Bytes in size, the channel conditions play the most important role in PER. However, for packets over 500 Bytes, $t=3$ performs 25 percentage points (pp) better than $t=1$ for either soil condition. Another interesting trend is seen in both environments where the error correction capability of seven bits achieves a high PRR and all but negates the effect of long packets (only increasing PER by $1 \mathrm{pp}$ ). On the other hand, for $t=3$, PRR has a strong dependence on packet size, ranging from $1 \%$ to over $20 \%$.

Detailed insight into how packets cross the channel using various error control protocols is observed in Fig. 3(a). Without retries or FEC (NoAF) and for packets over 300 Bytes, a packet has less than an $80 \%$ chance of making it to its 


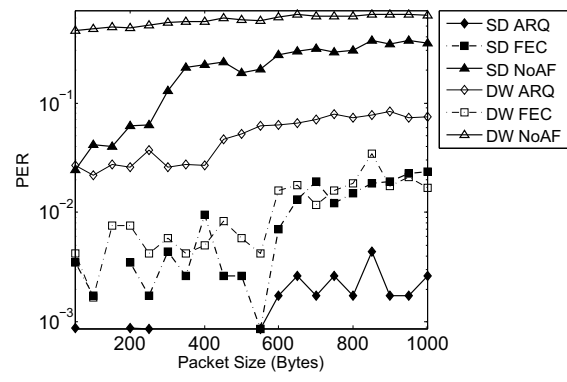

(a)

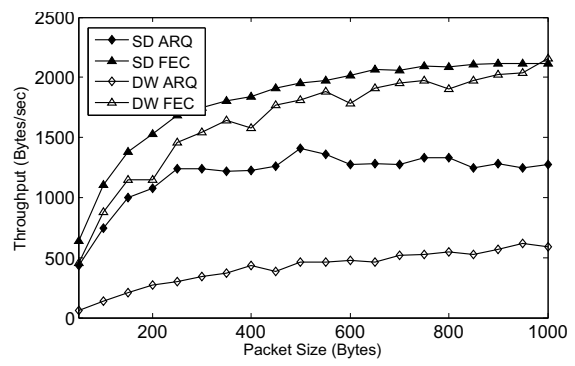

(b)

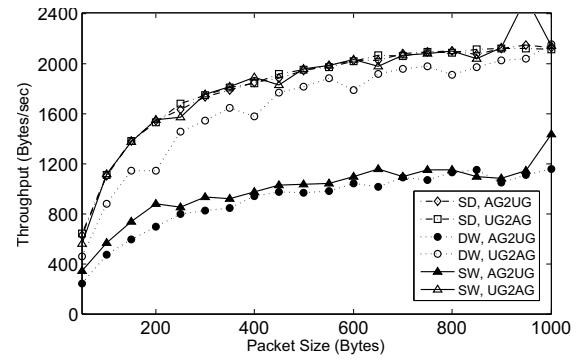

(c)

Fig. 3. (a)PER of various error control protocols, (b) UG2AG throughput in good (SD) and poor (DW) conditions using ARQ $(n=5)$ and FEC $(t=7)$, (c) Throughput vs. packet size for SD, SW, and DW.

destination due to bit errors. This is more pronounced for larger packets and in wet soil as $45 \%$ of the packets failed on their first attempt. In comparison, FEC provides better communication quality and is immune to channel conditions, i.e., PER changes by only \pm 1 pp between wet and dry environments. In good conditions (SD), the ARQ protocol had the best PER $(; 0.5 \%)$ with slight dependence on packet size, but in poor conditions (DW), there was a strong dependence on packet size as $2.5 \%$ of 50 Byte packets were lost compared to $8 \%$ of $1 \mathrm{kB}$ packets lost.

The throughput of ARQ and various FEC protocols can be seen in Fig. 3(b). Throughput is calculated based on payload size (not packet size). For a relatively good channel (SD), FEC is more tolerant of bit errors as packet size is increased, only dropping an additional $1 \mathrm{pp}$ of large $(1 \mathrm{kB})$ packets versus small packets (50 Bytes). For a poor channel (DW experiment), many retries are needed. Thus, the low latency of FEC (100ms to $500 \mathrm{~ms}$ for FEC versus $600 \mathrm{~ms}$ to $1500 \mathrm{~ms}$ for ARQ) yields a higher throughput, though it drops $1 \mathrm{pp}$ more packets. If a PER threshold of $3 \%$ were applied, ARQ would not be feasible for large packet sizes in wet conditions. An optimal throughput for ARQ in SD environment is achieved around 500 Bytes, but the other configurations achieved maximum throughput with packets $1 \mathrm{kB}$ in size.

The throughput of all three experiments is shown in Fig. 3(c) using the FEC protocol with $t=7$. In the wet experiments (DW and SW), the throughput for the AG2UG channel was half that of the UG2AG channel for all packet sizes: 300 Bytes $/ \mathrm{sec}$ to $600 \mathrm{Bytes} / \mathrm{sec}$ for 50 Byte packets and $1.2 \mathrm{kB} / \mathrm{sec}$ to $2.1 \mathrm{kB} / \mathrm{sec}$ for $1 \mathrm{kB}$ packets. The SD experiment, however, had a symmetric link. The DW UG2AG channel is $10 \%$ worse than the other UG2AG channels. This result is noteworthy when compared to the SNR results presented earlier. Despite the slightly worse channel quality compared to the DW experiment (which was reflected in the PER, not shown), the throughput of the SW experiment was better. This is due to the latency of the SW experiment as there was a $10 \mathrm{~ms}$ difference in latency between the shallow and the deep experiments.

\section{UG2AG and AG2UG Experiment Summary}

The results of these experiments provide insight into AG2UG and UG2AG channel characteristics, including the asymmetry between the UG2AG and AG2UG channels. Packet errors can be mitigated by using FEC and, to a lesser degree, ARQ. Packet size weakly affects PER when an ARQ or FEC protocol is implemented. Packet size determines the FEC strength needed to minimize dropped packets. A corrective ability of at least 7 bits is needed for packets over 600 Bytes in size. Latency (and therefore throughput) depends strongly on packet size and channel condition when an ARQ protocol is implemented. The UG2AG channel provides a better communication link than the AG2UG channel, most notable in wet conditions. Finally, it is observed that in poor conditions, an FEC protocol can make up for moist soil.

Sufficient data was gathered to represent the extreme UG2AG and AG2UG channels in a WUSN model, as will be discussed in Section IV.

\section{Mobile Data Harvesting In WUSNs}

As most of the work in WUSNs has been devoted to understanding underground channel properties, link- and networklevel considerations have not been analyzed yet. This is largely due to three major challenges: transmission range, energy conservation and cost. These challenges keep a traditional mesh WUSN from being feasible as the UG2UG wireless channel has a significantly lower range than UG2AG or AG2UG channels. To this end, a family of data harvesting protocols for WUSNs, based on mobile AG nodes, is presented. These protocols are evaluated in terms of delay tolerance, network lifetime and scalability.

\section{A. Connecting a WUSN with Mobile Sinks}

1) Network Setup: A practical WUSN consists of a set of UG nodes, $\mathcal{U}=\left\{u_{1}, u_{2}, \cdots\right\}$, and a set of mobile (AG) nodes, $\mathcal{M}=\left\{m_{1}, m_{2}, \cdots\right\}$. Thus, there are $|\mathcal{U}|+|\mathcal{M}|$ nodes in a network. When a mobile node is in communication range of an UG node, it may communicate with that UG node and this is the only way an UG node can communicate.

It is observed that the minimal soil event detection area (sensing range), $\alpha_{\text {min }}$, can be greater than the transmission range of UG2UG communication [2], [14]. Therefore, mobile 
sinks should be used to collect data by moving into close proximity to UG nodes. Hence, the number of UG nodes depends only on the event sensing area $\left(\alpha_{\min }\right)$, field size, and coverage requirement.

2) Mobile Nodes: Mobile sink nodes connect the network by collecting data from UG sensor nodes. Each mobile node navigates to a subset of UG nodes to collect their information, as indicated in Fig. 1. Thus, mobile nodes maintain a schedule by visiting each UG node at least once per $D_{T H}$. $D_{T H}$ is dependent on the specific WUSN application, but in general, the UG environment is slow changing [14]. As UG nodes are static, their location can be observed at deployment and routes need only be assigned once. It is sufficient to say at any given time, an UG node has a probability, $r$, of having a mobile node in its communication range as will be discussed next.

Mobile nodes are assumed to have sufficient energy for communication and in general have the ability to recharge themselves. This is a valid assumption for robotic, mobile nodes, where the energy cost of movement is over 10,000 times larger than the cost of wireless communication [1]. A robotic network will be assumed, giving full control of a node's movement, which assures travel between motes can be scheduled. Though mobile robots vary greatly, their properties do not affect the WUSN model so long as they are capable of meeting the scheduling requirements outlined above.

Mobile nodes are also assumed to have a reliable, long range connection to the Internet (such as a $3 \mathrm{G}$ cellular network) or other data subscriber (such as a center pivot irrigation controller). Thus, they do not need to buffer a large amount of data and the latency of transferring information from a mobile node to an interested party is assumed to be negligible.

The cost of mobile nodes includes the cost of maintenance and energy renewal. This cost can be approximated before a network is purchased, as the lifetime of a WUSN is limited by the UG lifetime.

3) Energy Management: Energy is a precious resource for UG nodes. Once buried, a node cannot easily harvest energy from its environment. UG nodes must choose network protocols carefully in order to efficiently use this energy while meeting the communication needs of the network.

Nodes operate in two modes: asleep and awake. While asleep, a mote only uses enough power, $P_{\text {sleep, }}$, to run a timer to wake itself up. When awake, the radio is on and it is capable of receiving and transmitting data as well as monitoring for events. Let the power used to transmit be $P_{T X}$ and receive be $P_{R X}$. It is assumed that if a node is awake and not transmitting, its radio is on and receiving.

The ratio of time a node spends awake to time spent asleep is its duty cycle, $\delta$. This period, $T_{s}$, is its sleep cycle. The total energy used per cycle, $E_{\text {cycle }}$, is defined as:

$$
\begin{aligned}
E_{\text {cycle }}= & P_{T X} \cdot T_{T X}+P_{R X} \cdot\left(T_{s} \cdot \delta-T_{T X}-T_{w}\right) \\
& +P_{\text {sleep }} \cdot(1-\delta) \cdot T_{s}+P_{w} \cdot T_{w}
\end{aligned}
$$

where $T_{w}$ is the time spent transitioning from the sleep state to awake state and back again, $P_{w}$ is the average power used during these transition periods and $T_{T X}$ is the time spent transmitting, which is bounded by the transition time and time the node is awake: $T_{T X} \leq \delta \cdot T_{S}-T_{w}$. If a node uses its maximum power, $P_{T X}$, when awake, the maximum duty cycle is:

$$
\delta_{\max }=\frac{E_{0}-T_{\text {life }} \cdot P_{\text {sleep }}}{T_{\text {life }} \cdot P_{T X}}
$$

as $P_{T X} \approx P_{T X}-P_{\text {sleep }} . T_{\text {life }}$ is the required network lifetime and $E_{0}$ is the initial energy contained in a mote's batteries.

\section{B. Data Harvesting Protocols}

Six protocols are introduced in Section IV-B2, which vary according to communication direction and FEC availability. The developed models are broken up into three parts: connectivity, protocol, and battery. The connectivity model determines the necessary conditions for events to be reported in a timely fashion and is constant for all protocols. The protocols examine the balance between time spent transmitting, receiving and sleeping. Finally, the battery model captures a long-term operating battery.

1) Connectivity Model: The logistics of getting a mobile node to each UG node is an issue outside the scope of this paper, but as mentioned previously, UG nodes cannot move, so mobile nodes can follow predetermined paths. The only requirement is that a mobile node come into range often enough to satisfy the latency threshold requirement, $D_{T H}$. It will be assumed that the travel time of mobile nodes is instant and a mobile node can only visit one UG node at a time. This assumptions means that $|\mathcal{M}| \mathrm{UG}$ nodes have a mobile node in range at all times ${ }^{1}$. Thus, the probability a mobile node is in range is $r$ :

$$
r=\frac{|\mathcal{M}|}{|\mathcal{U}|}=\frac{T_{s}}{D_{T H}}
$$

Let $q=r \cdot 100$ be used to represent number of mobile nodes needed per 100 UG nodes.

2) Protocol Models: The previous subsection defines the communication limits for connectivity, but does not explain how communication occurs. This subsection examines how best to use the energy of each mote using six protocols. Protocols were developed around two network constraints: communication direction and encrypting data using FEC.

Cases where two-way communication is not required present interesting opportunities. The first is that messages travel through the UG2AG channel better than the AG2UG channel. Limiting communication to only the UG2AG channel avoids the additional retries needed to receive control packets from AG nodes. An auxiliary observation is that UG nodes would not know if a mobile node is in range. This circumstance is not unlike two-way communication. With two-way communication, an UG mote would wake up and listen for a request message from a mobile sink. This requires turning the radio on, using almost as much energy to listen to the channel

\footnotetext{
${ }^{1}$ In practica, mobile node speed is not infinite but this condition can still hold: $|\mathcal{M}|^{\prime}$ mobile nodes are needed so that $|\mathcal{M}|$ are in range of UG nodes at any given them. This does not affect the model, only the total network cost, which can be minimized by using fast mobile nodes.
} 
as it would spend transmitting. It must listen for at least as long as the round trip time, $T_{R T T}$.

We refer to one-way UG2AG communication as announcement communication (AComm) as the UG mote simply announces its data. The first two protocols are announcement communication (AComm and AComm-FEC) protocols. These only transmit data once per sleep period, $T_{s}$. The next two also transmit only, but automatically repeat the transmission $n$ times per $T_{s}$. Let them be called AComm-AR ${ }^{2}$ and AComm-Hybrid, where AComm-Hybrid automatically sends with FEC encryption. When two-way communication is required, handshaking protocols are used: HComm-ARQ and HComm-Hybrid.

AComm (One-way communication with one attempt): An UG node without a receiver may only wake up, send its data, and go back to sleep on a periodic basis. This provides the lowest duty cycle possible, and thus, the longest network lifetime. As there is only one transmission attempt per packet, the chance for a successful transfer (when a mobile node is in range) is simply the success rate of data packets through the channel: $p_{s}^{d}$, which is a function of the payload size, $l_{D}$.

AComm-FEC (One-Way Communication with FEC): This is the AComm protocol where packets are FEC encrypted.

AComm-AR (One-Way Communication, with automatic retransmissions): Multiple attempts increase the chance of receiving a packet successfully. Thus, the actual sleep period, $T_{s}^{\prime}=T_{s} / n$, where $n$ is the largest number of attempts such that (1) holds true. The chance for a successful transfer (when a mobile node is in range) is $p_{s}=1-\left(1-p_{s}^{d}\right)^{n}$.

AComm-Hybrid (One-Way Communication with automatic FEC retransmissions): This protocol sends packets $n$ times with FEC encryption.

The AComm protocols attempt to send data without knowing if there is a mobile node in range. However, this may not be optimal, nor is it possible if two-way communication is required. In these cases, UG nodes must listen to the channel before sending data.

For two-way communication, a handshaking protocol is employed. Since a mobile node does not know when the UG node will be awake, it must send request packets every $T_{r q}$ period until it receives data or runs out of attempts. Mobile nodes must wait up to $T_{s}$ time at each UG node to wake them up.

There is a limit to the number of data transmission attempts, $N$. The mobile node is on a schedule and may not linger at a single UG node for too long. The UG node must also go to sleep, otherwise it will run out of energy attempting to send a packet too many times. Accordingly, the request period is bounded by:

$$
T_{R T T} \leq T_{r q} \leq \delta \cdot T_{s}
$$

where $T_{R T T}$ is the time taken to send a request packet and receive data. If $T_{r q}=\delta \cdot T_{s}$, only one request attempt can be made per sleep cycle. As $T_{r q}$ period decreases, the chance of the UG node correctly receiving a request message goes

\footnotetext{
${ }^{2}$ Retransmissions are automatic (AR) but are not requested (not ARQ)
}

up, as does $N$. Accordingly, the following two handshaking protocols are employed as explained next.

HComm-ARQ (Two-Way Communication with handshaking): Two-way communication requires a mobile sink to send out request packets as it waits for the UG node to wake up. After waking up and hearing a request, the UG node will send its data and wait for an acknowledgment. The probability that data is successfully transferred depends on request, data, and ACK packets being received correctly with $N$ attempts:

$$
p_{s}=1-\left(1-p_{s}^{c} \cdot p_{s}^{d} \cdot p_{s}^{c}\right)^{N}
$$

where $p_{s}^{c}$ is the probability that a single control packet from AG to UG is received correctly and $N$ is the retry limit.

HComm-Hybrid (Two-way communication with handshaking and FEC): This is the HComm-ARQ protocol with FEC-encrypted packets.

3) Battery Model: A simple, constant current loss battery model is used to evaluate each of these protocols [15]. Two AA batteries with a 10 year lifetime leak approximately $68.4 \mu \mathrm{W}$ of power. Due to the extremely low duty cycle, this leak power can be included within the sleep power creating an effective $P_{\text {sleep }}$ of $107.4 \mu \mathrm{W}$ when Mica2 motes are used [16].

\section{Performance Evaluation}

\section{A. Semi-Empirical Evaluation Models}

The six protocols described in Section IV-B2 are evaluated using the data collected in Section III and the protocol constraints described in Section IV-B2. Payload size $\left(l_{D}\right)$ and channel conditions $\left(p_{s}^{d}, p_{s}^{c}\right)$ are varied to capture changes in network lifetime, throughput and reception rate for various delay thresholds $\left(D_{T H}\right)$ and mobile to UG node ratios $(r)$.

Along with (1) and the packet reception rates discussed with each protocol, the lifetime, $T_{\text {life }}$, and the throughput can be found as follows for a given delay tolerance, $D_{T H}$, and $r$, number of mobile to UG nodes. Given the sleep period $T_{s}=$ $D_{T H} \cdot r$, the lifetime is found as:

$$
T_{\text {life }}=\frac{E_{0}}{\delta \cdot P_{\text {awake }}+(1-\delta) \cdot P_{\text {sleep }}}
$$

where $E_{0}$ is the initial energy, $\delta$ is the duty cycle, and the awake power is given as

$$
P_{\text {awake }}=r \cdot\left[\gamma \cdot P_{T X}+(1-\gamma) \cdot P_{R X}\right]+(1-r) \cdot P_{R X}
$$

where

$$
\gamma=\frac{T_{T X}}{T_{T X}+T_{R X}}=\frac{T_{T X}}{T_{\text {awake }}}
$$

is the ratio between time spent transmitting over the awake time. For the AComm protocols, $\gamma=1$ as the UG motes only transmit. Accordingly, the duty cycle can be written as:

$$
\delta=\frac{D_{T H} \cdot\left(\frac{E_{0}}{T_{\text {life }}}-P_{\text {sleep }}\right)+\left(P_{R X}-P_{T X}\right) \cdot T_{T X}}{D_{T H} \cdot\left(P_{R X}-P_{\text {sleep }}\right)}
$$

The time spent transmitting, $T_{T X}$ in (7) and (9) is found as

$$
T_{T X}=T_{r q} \cdot\left(\frac{1}{p_{s}^{d}}+\frac{\left(1-p_{s}^{d} \cdot p_{s}^{c}\right)\left(1-p_{s}^{d} \cdot p_{s}^{c}\right)^{N}}{p_{s}^{d} \cdot p_{s}^{c}}\right),
$$




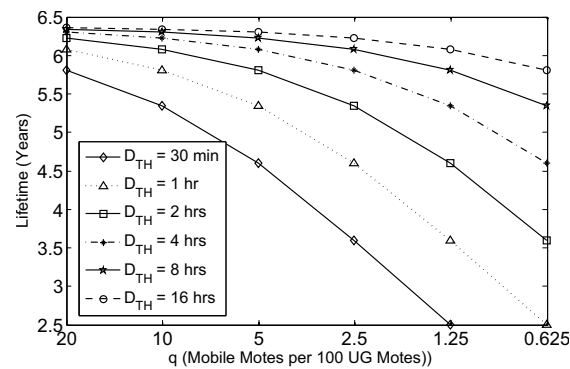

(a)

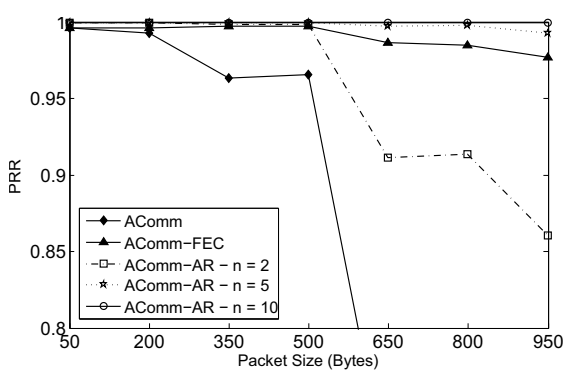

(b)

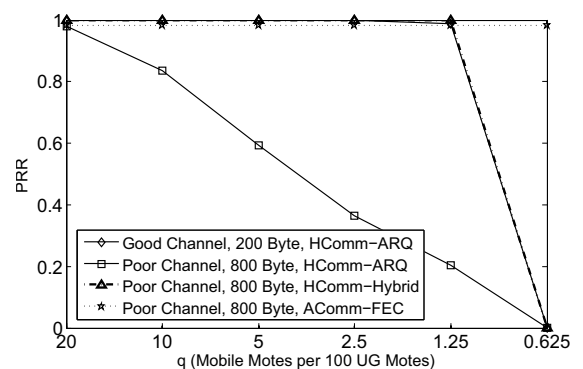

(c)

Fig. 4. (a) Network lifetime in terms of $q$ and $D_{T H}$ for AComm and AComm-FEC, (b) PRR of the announcement protocols in good channel conditions, (c) PRR using HComm-ARQ and HComm-Hybrid (FEC) in various conditions and packet sizes with $D_{T H}=2$ hours.

TABLE II

THE VAlues USED TO EVAlUATE THE NETWORK PROTOCOLS. POWERS TAKEN FROM THE MICA2 DATA SHEET [16] AND [17].

\begin{tabular}{llll}
\hline Symbol & Property & Value & Unit \\
\hline$T_{l i f e}$ & Network Lifetime & 5 & Years \\
$E_{0}$ & Initial Energy (2 AA batteries) & 21.6 & $\mathrm{~kJ}$ \\
$P_{T X}$ & Power consumption while transmitting & 66 & $\mathrm{~mW}$ \\
$P_{R X}$ & Power consumption while receiving & 57 & $\mathrm{~mW}$ \\
$P_{\text {sleep }}$ & Power used in sleep \& battery discharge & 107.4 & $\mu \mathrm{W}$ \\
$P_{w}$ & Power while waking up or going to sleep & 45.3 & $\mathrm{~mW}$ \\
$T_{w}$ & Transition time to wake up + go to sleep & 3.6 & $\mathrm{~ms}$ \\
\hline
\end{tabular}

where $T_{r q}$ is the request period according to (4), $p_{s}$ is the overall success rate where $p_{s}^{c}$ and $p_{s}^{d}$ are the success rate of control and data packets respectively, and $N$ is the allowed number of retries, $N$, as given by

$$
N=\left.\frac{\delta \cdot T_{s}}{T_{r q}}\right|_{T_{R T T} \leq T_{r q} \leq \delta \cdot T_{s}}
$$

Finally, the throughput is found as:

$$
\text { Throughput }=\frac{l_{D}}{T_{s}} \cdot\left(1-p_{s}\right)
$$

For AComm-AR and AComm-Hybrid, $N$ is $n, T_{R T T}$ becomes $T_{T X}$, the time it takes to transmit a data packet one way. Thus, (10) only applies to the HComm protocols. To simplify the analysis of the two-way communication, the transition time $\left(T_{w}\right)$ was assumed to be negligible. This increases the lifetime of the network by less then $1 \%$, which is an acceptable inaccuracy.

The Mica2 motes used in the experiments are modeled based on the parameters in Table II. These models are valid as they address the problems of WUSN lifetime and scalability, represent a real-world network as empirically collected data is used in their evaluation, and account for all energy usage.

\section{B. Network Lifetime}

In protocols AComm and AComm-FEC, an UG node can wake up as often as needed to satisfy the delay threshold, $D_{T H}$, which is related to the number of mobile of nodes in the network. This relation is shown in Fig. 4(a). For $D_{T H}=$ $30 \mathrm{~min}$, it can be seen that increasing the ratio of mobile nodes to UG nodes by a factor of 20 increases the network lifetime by a factor of two. With more mobile nodes, UG nodes can wake up less often since there is a higher probability that a mobile node will be available when it wakes up. The impact of mobile nodes on the lifetime is less pronounced when the delay threshold is higher. A longer $D_{T H}$ means the UG node can have a lower duty cycle. The maximum network lifetime (for a long $D_{T H}$ and high $r$ ) yields a duty cycle near zero, where lifetime is mainly determined by the sleep current and battery leakage.

\section{Packet Reception Rate}

The effects of packet size and protocol type on packet reception rate (PRR) is shown in Fig. 4(b). Both the AComm and AComm-AR with $n=2$ have PRRs less than $90 \%$ for packet sizes greater than 500 bytes, while AComm-FEC and AComm-AR with $n \geq 5$ have PRR over $98 \%$ for packet sizes up to $1 \mathrm{kB}$. The PRR in the good channel was less than $1 \mathrm{pp}$ worse for AComm-FEC and AComm-AR and $3 \mathrm{pp}$ worse for AComm. AComm is equal to AComm-AR with $n=1$, as is AComm-FEC to AComm-Hybrid. AComm-FEC is the best protocol to use as it achieves high reliability (99.5\% to $98 \%$ PRR for packets 50 to 950 Bytes) and long network lifetimes. AComm-Hybrid may be used when packets over 800 Bytes need to be received with a success rate over $98 \%$ in good or poor channels. AComm-AR increases reliability when an FEC protocol is not available.

HComm-ARQ and HComm-Hybrid differ from the previous protocols in that UG nodes wake up and listen to the channel, only transmitting when requested. This allows for two-way communication, but means listening idly to the channel most of the time (1-r part of the time). When a mobile node is in range, it has time for $N$ attempts. The longer an UG node is awake, the more retransmissions are possible, increasing the chance of successful communication.

The number of retransmissions allowed for a lifetime requirement of 5 years can be seen in Table III, where configurations with blank entries are not feasible. Data from the dry UG2AG channel was used for this table, but a worse PER (e.g., wet data) affects $N$ by less than 5\%. For example, for $q=2$ (two mobile nodes per $100 \mathrm{UG}$ nodes), there are not enough mobile nodes in the network to collect all the data in 30 minutes. If one hour delay is allowed, then , two mobile nodes per $100 \mathrm{UG}$ nodes are sufficient whereas only 


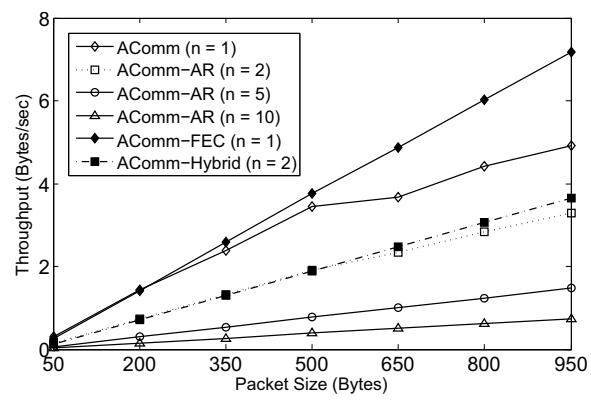

(a)

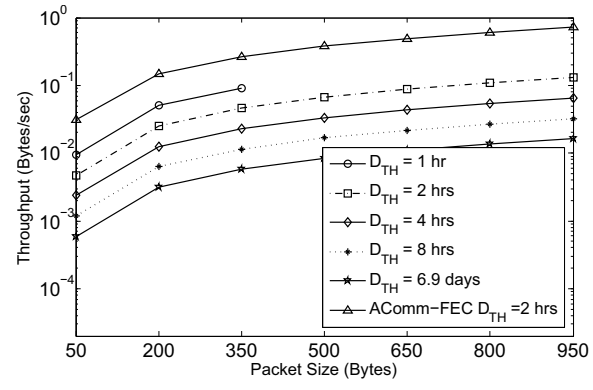

(b)

Fig. 5. Throughput results: (a) $T_{l i f e}=5$ years using AComm protocols and (b) Using HComm-Hybrid with $q=2.5$ mobile motes per 100 UG motes.

TABLE III

NUMBER OF RETRANSMISSIONS ALLOWED PER PACKET, $N$, TO MEET A LIFETIME REQUIREMENT OF 5 YEARS (UG2AG CHANNEL).

\begin{tabular}{l|llllll}
\hline & \multicolumn{6}{|c}{ Delay Threshold $\left(D_{T H}\right)$} \\
$q$ & $30 \mathrm{~min}$ & $1 \mathrm{hr}$ & $2 \mathrm{hrs}$ & $4 \mathrm{hrs}$ & $8 \mathrm{hrs}$ & $16 \mathrm{hrs}$ \\
\hline 20 & 5 & 10 & 21 & 43 & 86 & 173 \\
10 & 2 & 5 & 10 & 21 & 43 & 86 \\
5 & 1 & 2 & 5 & 10 & 21 & 43 \\
2 & & 1 & 2 & 5 & 10 & 21 \\
1.25 & & & 1 & 2 & 5 & 10 \\
0.625 & & & & 1 & 2 & 5 \\
\hline
\end{tabular}

one attempt is possible. If the delay threshold is increased to four hours, $q$ may be varied so that there are between five and hundreds of UG nodes for every mobile node, though decreasing $q$ reduces the time spent at each UG node, indicated by a lower value of $N$. The delay threshold has the most impact on how many transmissions can be afforded during each sleep period and PRR.

The PRR performance of duplex protocols is shown in Fig. 4(c), where AComm-FEC is also shown as a reference. When using FEC, only one mobile node is sufficient for every eighty UG to maintain a network with a good packet reception rate (98\%). When FEC is not available and channel conditions are poor, control packets cannot get through, and many additional retries are necessary for a packet to be received correctly, e.g., over 90 retries to achieve $80 \%$ PRR.

\section{Throughput}

Throughput is another consideration for efficient data harvesting. In Fig. 5(a), the strong dependence of throughput on packet size for the AComm protocols is shown. A linear relationship can be seen for all protocols, though AComm saturates after 500 bytes. This is due to the PRR sharply decreasing with packets greater than 500 Bytes as shown in Fig. 4(b). For 50 Byte and 200 Byte packets, impacts of FEC are negligible as both AComm and AComm-FEC are within $2 \mathrm{pp}$ of each other, even in poor (wet) conditions. As transmission rate is determined by network lifetime, only packet size may be varied to adjust the throughput of an UG node. FEC is essential to high throughput, especially when no retries are available. Multiple attempts greatly increase the chance of a packet being received correctly, as seen in Section
III. AComm-AR and AComm-Hybrid take advantage of excess energy by retransmitting messages as often as possible while maintaining a network lifetime, $T_{\text {life }}=5$ years is shown. In a simplex WUSN, AComm-AR and AComm-Hybrid should only be implemented if PRR requirements cannot be met by using AComm-FEC, as throughput falls by a factor of $n$.

The throughput of the handshaking protocols with respect to packet size and delay tolerance is shown in Fig. 5(b). When there are 2.5 mobile nodes for every UG node, throughput is maximized by relaxing the delay tolerance to two hours as there is not enough time to send packets longer than 350 Bytes every hour. Network throughput is inversely proportional to $D_{T H}$, though there is a limit to how short $D_{T H}$ can be while still maintaining a connected network. For the same $D_{T H}$ and $q$, AComm-FEC has a throughput between three and five times higher than HComm-Hybrid. This is due to the asymmetry between the UG2AG channel and the AG2UG channel as discussed in Section III-B. Moreover, sending data requires less time than the time it takes to receive a request, switch the radio over to transmit and then transmit the packet.

\section{E. Discussions}

Each protocol has its strengths and weaknesses as listed in Table IV, which serves as a guide when choosing a WUSN protocol. In every case, FEC should be used, if available. FEC is able to provide a reliable communication link and is immune to the changes in the soil environment.

Intuitively, the dynamic properties of the channel suggest that an UG node may be able to change its protocol based on changes in the soil moisture. The empirical evaluations reveal that FEC reduces the effects of soil moisture on communication at the cost of without significant overhead in packet size or energy (as decryption is performed at the mobile nodes). Implementations, where FEC is not available, may need to vary the attempts $(n)$ used in AComm-AR based on soil moisture, e.g., retransmit a packet more often when the soil is wet. Changes in UG nodes with respect to soil moisture may not be pronounced with HComm-ARQ, except letting the AG node send multiple request messages at once, which does not affect the duty cycle of the UG node nor the lifetime of the network. Overall, FEC is highly encouraged due to these issues. 
TABLE IV

POINTS TO CONSIDER WHEN CHOOSING A WUSN PROTOCOL

\begin{tabular}{|c|c|c|}
\hline Protocol & Strengths & Weaknesses \\
\hline AComm & $\begin{array}{lrr}\text { Long } & \text { NW } & \text { Lifetime, } \\
> & 99 \% & \text { PRR } \\
\left(l_{D} \leq 50 \text { Byte }\right) & \end{array}$ & $\begin{array}{l}\text { Poor PRR }\left(l_{D} \geq 200 \text { Byte) }\right. \\
\text { one-way communication }\end{array}$ \\
\hline $\begin{array}{l}\text { AComm- } \\
\text { FEC }\end{array}$ & $\begin{array}{l}\text { Long NW Lifetime, High- } \\
\text { est Throughput, }>98 \% \\
\text { PRR for sizes up to } 1 \mathrm{kB}\end{array}$ & One-way communication \\
\hline $\begin{array}{l}\text { AComm- } \\
\text { AR }\end{array}$ & $\begin{array}{l}\text { High reliability without } \\
\text { FEC }\end{array}$ & $\begin{array}{l}\text { Low throughput, one-way com- } \\
\text { munication }\end{array}$ \\
\hline $\begin{array}{l}\text { AComm- } \\
\text { Hybrid }\end{array}$ & Highest PRR & $\begin{array}{l}\text { Lower throughput than } \\
\text { AComm-FEC, one-way } \\
\text { communication }\end{array}$ \\
\hline $\begin{array}{l}\text { HComm- } \\
\text { ARQ }\end{array}$ & $\begin{array}{l}\text { Two-way communication, } \\
\text { simple to implement, no } \\
\text { FEC }\end{array}$ & $\begin{array}{l}\text { Poor PRR in poor channels, } \\
\text { Low throughput }\end{array}$ \\
\hline $\begin{array}{l}\text { HComm- } \\
\text { Hybrid }\end{array}$ & $\begin{array}{l}\text { Two-way communication, } \\
\text { High throughput and PRR } \\
\text { in poor channel }\end{array}$ & $\begin{array}{l}\text { Requires FEC support, } \\
\text { Throughput is } 20 \% \text { of AComm- } \\
\text { FEC }\end{array}$ \\
\hline
\end{tabular}

\section{CONCLUSiOnS AND FUtURE WORK}

A practical wireless underground sensor network model for mobile data harvesting and a family of protocols are presented and are shown to meet the preconditions of having no permanent aboveground fixtures and being scalable to large areas. Evaluations with network lifetime suggest that unattended operation of 5 or more years is possible with the apropriate selection of error control protocols and mobile network architectures. WUSNs are applicable in delay tolerant situations where event delay is related to throughput and the ratio of mobile nodes to UG nodes.

These protocols are the first to be presented, which address network lifetime and allow for large field deployments. The issues of connectivity and channel quality were addressed to assure high packet reception rates. Semi-empirical models are developed to evaluate the protocols based on empirically gathered data in a large-scale agriculture testbed.

While the reliance on mobile nodes precludes certain WUSN applications (such as intruder detection), it allows for continuous field monitoring, a need that has not been fulfilled in precision agriculture and sports field maintenance yet. As a future work, the developed WUSN protocols will be empirically evaluated with testbed experiments. Integration with actual mobile nodes is also a non-trivial activity that would need to be done before a real experiment were to be performed.

These results suggest that multi-hop UG networks are not feasible for large-scale applications with respect to network lifetime and scalability. In order for multi-hop WUSNs to be feasible, the transmission range of UG nodes would need to be extended to feasible distances. Moreover, these ranges should be attainable while using low transmit powers so as to operate on a fixed amount of energy for extended periods of time, which is long enough to make a deployment cost effective.

In this paper, WUSNs have been shown to be practical and useful in delay tolerant situations by using mobile nodes to gather data while extending network lifetime and scalability. Through an empirical understanding of wireless underground channel reliability, a family of protocols was developed and evaluated in terms of packet delivery success, delay tolerance and network lifetime.

\section{REFERENCES}

[1] I. F. Akyildiz, W. Su, Y. Sankarasubramaniam, and E. Cayirci, "Wireless sensor networks: a survey," Computer Networks, vol. 38, no. 4, pp. 393 -422 , mar. 2002.

[2] M. C. Vuran and I. F. Akyildiz, "Channel model and analysis for wireless underground sensor networks in soil medium," Physical Communication, vol. 3, pp. 245-254, Dec. 2010.

[3] I. F. Akyildiz and E. P. Stuntebeck, "Wireless underground sensor networks: Research challenges," Ad Hoc Networks, vol. 4, no. 6, pp. 669 - 686, Nov. 2006.

[4] Z. Sun and I. Akyildiz, "Connectivity in wireless underground sensor networks," in Proc. IEEE SECON '10, Boston, MA, June 2010.

[5] R. C. Shah, S. Roy, S. Jain, and W. Brunette, "Data MULEs: modeling and analysis of a three-tier architecture for sparse sensor networks," Ad Hoc Networks, vol. 1, no. 2-3, pp. 215 - 233, Sept. 2003.

[6] "CIA the world factbook: GDP - composition by sector," https://www.cia.gov/library/publications/the-worldfactbook/fields/2012.html.

[7] V. Adamchuk, J. Hummel, M. Morgan, and S. Upadhyaya, "On-thego soil sensors for precision agriculture," Computers and Electronics in Agriculture, vol. 44, no. 1, pp. 71 - 91, July 2004.

[8] R. Cardell-Oliver, M. Kranz, K. Smettem, and K. Mayer, "A reactive soil moisture sensor network: Design and field evaluation," International Journal of Distributed Sensor Networks, vol. 1, pp. 149-162, 2005.

[9] A. R. Silva and M. C. Vuran, "Communication with aboveground devices in wireless underground sensor networks: An empirical study," in Proc. IEEE ICC 2010, Cape Town, South Africa, May 2010.

[10] M. Vuran and I. Akyildiz, "Cross-layer packet size optimization for wireless terrestrial, underwater, and underground sensor networks," in Proc. IEEE INFOCOM '08, Phoenix, AZ, April 2008.

[11] X. Dong and M. C. Vuran, "A channel model for wireless underground sensor networks using lateral waves," in Proc. IEEE GLOBECOM '11, Houston, TX, Dec. 2011.

[12] M. Vuran and I. Akyildiz, "XLP: A cross-layer protocol for efficient communication in wireless sensor networks," IEEE Trans. Mobile Computing, vol. 9, no. 11, pp. 1578 -1591, Nov. 2010.

[13] A. R. Silva and M. C. Vuran, "(CPS $)^{2}$ : integration of center pivot systems with wireless underground sensor networks for autonomous precision agriculture," in Proc. ACM/IEEE Int. Conf. Cyber-Physical Systems, Stockholm, Sweden, Apr. 2010, pp. 79-88.

[14] D. Corwin and S. Lesch, "Characterizing soil spatial variability with apparent soil electrical conductivity: I. survey protocols," Computers and Electronics in Agriculture, vol. 46, no. 1-3, pp. 103 - 133, Mar. 2005.

[15] P. Arora, R. E. White, and M. Doyle, "Capacity fade mechanisms and side reactions in lithium-ion batteries," Journal of The Electrochemical Society, vol. 145, no. 10, pp. 3647-3667, Oct. 1998.

[16] Crossbow, "MPR-MIB users manual," www.memsic.com.

[17] H. Dubois-Ferrire, R. Meier, L. Fabre, P. Metrailler, E. Shockfish, S. Epfl, and S. Sa, "TinyNode: a comprehensive platform for wireless sensor network applications," in Proc. IPSN '06 (Poster). ACM Press, apr. 2006, pp. 358-365. 